\title{
Amiodarone and risk of liver cirrhosis: a nationwide, population-based study
}

This article was published in the following Dove Press journal:

Therapeutics and Clinical Risk Management

\author{
Ching-Hui Huangl-3,* \\ Ya-Yun Lai ${ }^{4, *}$ \\ Yu-Jui Kuo ${ }^{4,5}$ \\ Su-Ching Yang ${ }^{6}$ \\ Yu-Jun Chang ${ }^{7}$ \\ Kuo-Kuan Chang ${ }^{8}$ \\ Wen-Kang Chen ${ }^{4}$ \\ 'Division of Cardiology, Department of \\ Internal Medicine, Changhua Christian \\ Hospital, Changhua, Taiwan; ${ }^{2}$ Department \\ of Beauty Science, Graduate Institute \\ of Beauty Science Technology, Chienkuo \\ Technology University, Changhua, \\ Taiwan; ${ }^{3}$ School of Medicine, College of \\ Medicine, Kaohsiung Medical University, \\ Kaohsiung, Taiwan; ${ }^{4}$ Department of \\ Applied Cosmetology, National Tainan \\ Junior College of Nursing, Tainan, Taiwan; \\ ${ }^{5}$ Department of Traditional Chinese \\ Medicine, Tainan Municipal Hospital \\ (Managed by Show Chwan Medical Care \\ Corporation), Tainan, Taiwan; ${ }^{6}$ Department \\ of Nursing, National Tainan Junior College \\ of Nursing, Tainan, Taiwan; ${ }^{7}$ Epidemiology \\ and Biostatistics Center, Changhua \\ Christian Hospital, Changhua, Taiwan; \\ ${ }^{8}$ Department of Hepatogastroenterology, \\ Tainan Municipal Hospital (Managed by \\ Show Chwan Medical Care Corporation), \\ Tainan, Taiwan \\ *These authors contributed equally \\ to this work
}

Correspondence: Kuo-Kuan Chang Department of Hepatogastroenterology, Tainan Municipal Hospital (Managed by Show Chwan Medical Care Corporation), No. 670, Chongde Rd., East Dist., Tainan City 70I, Taiwan

Tel +886 62609926

Fax +886 62255308

Email kchang0902@yahoo.com.tw

Wen-Kang Chen

Department of Applied Cosmetology,

National Tainan Junior College of Nursing, 78,

Sec 2, Minzu Road, Tainan City 700, Taiwan

Tel +886 62609926

Fax+886 62255308

Email cwk@mail.ntin.edu.tw
Background: Liver cirrhosis is an uncommon but not rare side effect of amiodarone-induced hepatotoxicity. Patients with hepatitis B virus and hepatitis $\mathrm{C}$ virus infections are at a high risk for developing liver cirrhosis. However, the relationship between this treatment and risk of liver cirrhosis in high-risk chronic hepatitis $\mathrm{B}$ and chronic hepatitis $\mathrm{C}$ patients is unknown. Patients and methods: The present study identified amiodarone users $(\mathrm{N}=8,081)$ from the Taiwan National Health Insurance Research Database from 1997 through 2013. A total of 32,324 subjects with age, comorbidities, gender, and index date-matched non-amiodarone users were selected as controls (non-amiodarone cohort). The incidences of cumulative liver cirrhosis were compared between cohorts. Stratified Cox's regression hazard models were used to assess possible comorbidity-attributable risks for liver cirrhosis.

Results: The amiodarone cohort had a nonsignificant risk of liver cirrhosis compared with the non-amiodarone cohort, with a HR of 1.17 (95\% CI: 0.93-1.47; $P=0.1723)$. Patients with specific comorbid diseases, including type 2 diabetes mellitus, chronic hepatitis B, chronic hepatitis $\mathrm{C}$, and heart failure, were probably at a high risk of developing liver cirrhosis. The use of statins was associated with a significant $42 \%$ reduction in the risk of liver cirrhosis.

Conclusion: Patients in the amiodarone cohort had no excess risk of liver cirrhosis compared with patients in the non-amiodarone cohort. Long-term surveillance for liver toxicity in highrisk patients with amiodarone treatment is suggested.

Keywords: amiodarone, liver cirrhosis, Taiwan, hepatitis

\section{Introduction}

Amiodarone is a class III antiarrhythmic drug widely used for ventricular and supraventricular arrhythmias in patients with heart disease, including left ventricular dysfunction. ${ }^{1,2}$ Amiodarone exerts negligible negative inotropic activity and causes a low rate of ventricular proarrhythmia, rendering this drug advantageous for use in patients with heart failure. ${ }^{3}$ The latest 2016 guidelines for the management of atrial fibrillation recommended that for patients with heart failure, amiodarone is the drug of choice for maintaining sinus rhythm. ${ }^{4,5}$ Liver disease is a common sequela of advanced heart failure, and it ranges from mild reversible liver injury to hepatic fibrosis and, in its most severe form, cardiac cirrhosis. ${ }^{6}$ Hepatic dysfunction, whether primary or resulting from cardiac disease, can significantly alter the pharmacokinetics of many medications. Unfortunately, in hepatic dysfunction, serum drug levels and target effects are often unpredictable, and they show no good correlation with the specific cause of liver disease, its severity or level of liver function. ${ }^{7}$

Hepatotoxicity is a recognized amiodarone complication that is commonly mild with delayed onset. However, liver cirrhosis is an under recognized side effect of this amiodarone-induced hepatotoxicity. ${ }^{8}$ Moreover, with the high prevalence of 
hepatitis B and C in Taiwan, patients with hepatitis B virus (HBV) and hepatitis $\mathrm{C}$ virus (HCV) infections are at a high risk for developing liver cirrhosis. Nonetheless, the relationship between amiodarone treatment and risk of liver cirrhosis in high-risk patients is unknown. Since liver injury from amiodarone is uncommon but not rare. ${ }^{9}$ Large-scale population and long-term follow-up database provides good opportunity to evaluate the association.

Therefore, we aimed to determine the incidence of liver cirrhosis between amiodarone-treated and non-amiodaronetreated groups using data from the National Health Insurance Research Database (NHIRD). We also aimed to ascertain whether HBV or HCV infection exerts a detrimental effect on amiodarone users with liver cirrhosis.

\section{Materials and methods Data sources}

Taiwan's National Health Insurance (NHI) system has covered more than $99 \%$ of its 23 million inhabitants since 2000 . This study used two subsets of the NHIRD (Longitudinal Health Insurance Database [LHID] 2000 and LHID 2005) for vertical medical records from 1997 to 2012. The LHID 2000 contains the complete original claims data of 1 million insured individuals who were randomly sampled from the NHIRD registry in 2000. And the LHID 2005 also contains the 1 million insured individuals who were randomly sampled from the NHIRD registry in 2005. The NHIRD is composed of anonymous secondary data released to the public for research purposes. All patients' data were encrypted using the same encryption algorithm to cross-link the data while protecting the privacy of the patients. The study randomly selected 2 million insured individuals data, which contain health care data including date of birth, gender, income, dates of clinical visits, diagnostic codes, details of prescriptions, procedures, surgeries, expenditures amounts, and others. All diseases were identified based on the International Classification of Diseases, Ninth Revision, Clinical Modification (ICD-9-CM) codes in the NHIRD. The data accessed do not hold identifying patient information. This study was approved by the National Institutes of Health and the Tainan Municipal Hospital.

\section{Study subjects}

A flowchart of the subject selection process is shown in Figure 1. Considering hepatotoxicity of amiodarone complication is usually with delayed onset, we defined subjects who received amiodarone medications longer than 90 days within 1 year were eligible for inclusion in amiodarone cohort. The study included subjects with age ranged within 40-100 years, and those who were able to meet the inclusion criteria for amiodarone cohort and had received amiodarone medications from 1997 to 2010. Subjects who received amiodarone medication $<90$ days within 1 year were excluded; those aged $<40$ or $>100$ years; those with incomplete demographic data; those who received his/her first amiodarone medication after 2010; and those with a history of liver cirrhosis, hepatotoxicity or liver injury before the first amiodarone medication were excluded from the amiodarone cohort. Follow-ups were initiated 1 year after the first amiodarone medication, which was also designated as the index date. Subjects who had not taken amiodarone before were selected from the database and were treated as the non-amiodarone cohort. Those with a history of liver cirrhosis, hepatotoxicity or liver injury before the index data were excluded from non-amiodarone cohort. Prescribed use of other medicines, such as cardiovascular drugs, diabetic drugs, statins, interferons, and nucleoside analogs for chronic hepatitis $\mathrm{B}(\mathrm{CHB})$, ribavirin or peginterferon for chronic hepatitis $\mathrm{C}(\mathrm{CHC})$, between the index date and 1 year after the index day was also considered. We searched $\mathrm{CHB}$ patients receiving treatment with interferons, lamivudine, adefovir, telbivudine, and entecavir. Taiwan's NHI program has strict regulations regarding reimbursement for nucleoside analogs. Reimbursement for nucleoside analogs requires patients to fulfill certain criteria, such as twice-elevated serum alanine aminotransferase $([\mathrm{ALT}] \geq 2 \times)$ and elevated HBV DNA titer $(>2,000 \mathrm{IU} / \mathrm{mL}) .{ }^{10} \mathrm{We}$ also searched $\mathrm{CHC}$ patients receiving treatment with ribavirin and peginterferon. The CHC patients fulfilled the treatment criteria such as positive anti-HCV, two values (3 months apart) of abnormal ALT levels higher than two times of upper limit of normal and liver biopsy proved hepatic fibrosis could be covered by the reimbursement policy. ${ }^{11}$ All medication prescription records contained dates of order, dosage, and number of days prescribed for each dispensed drug. The study explored the use of the drug for a period of 1 year after the index date. Subject models were individually matched at a ratio of $4: 1$, whereas patients in the amiodarone cohort were matched for age, gender, propensity scores for comorbidities and medication, and index date. Non-amiodarone patients who were diagnosed with liver cirrhosis in the first year after the index date were also excluded. All participants were thoroughly observed until the following cases occurred: 1) diagnosis of liver cirrhosis, 2) withdrawal from the NHI program, 3) death, or 4) end of experimental period (2012). The date of withdrawal from the NHI program has been considered as a 


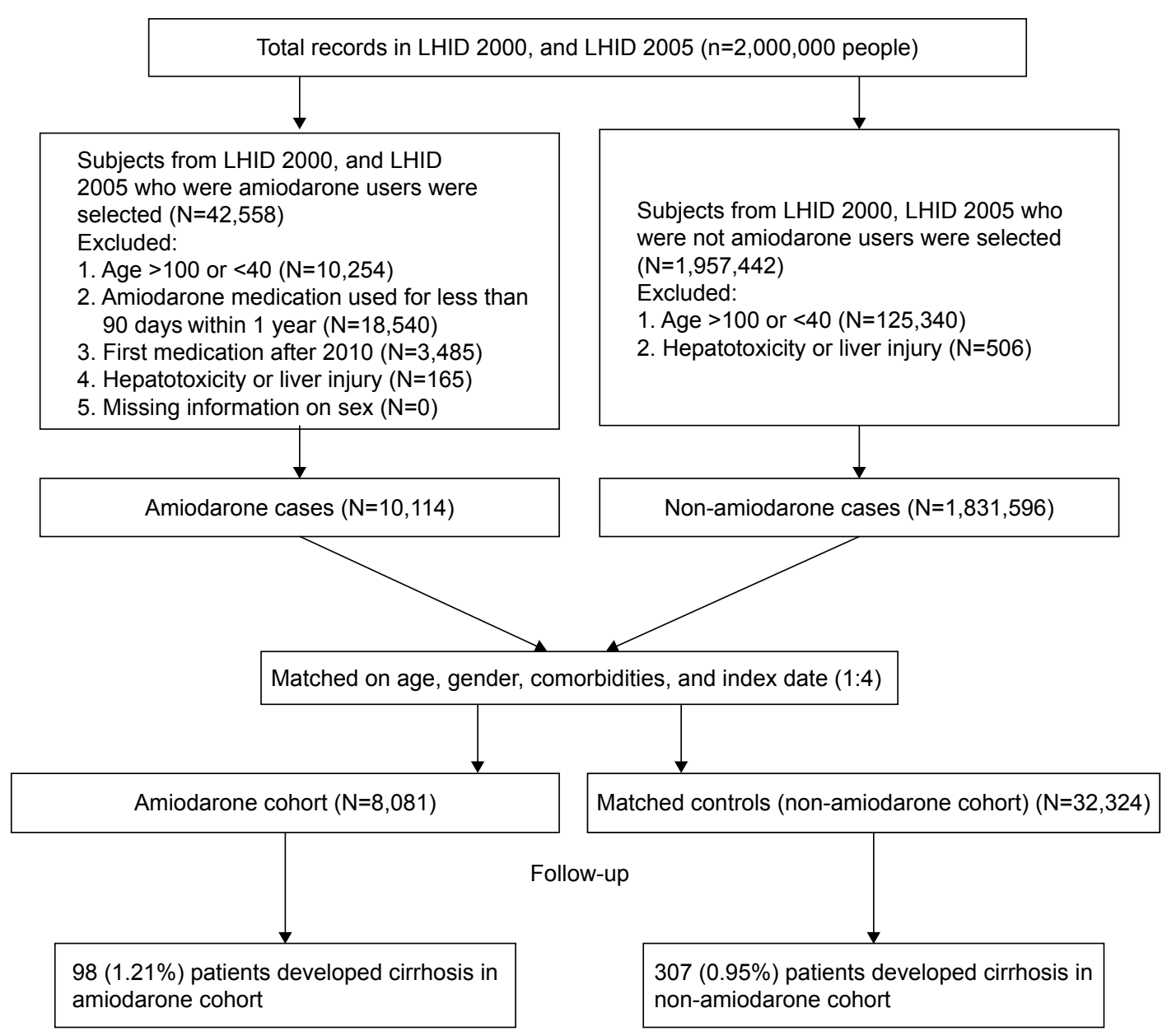

Figure I Study flowchart.

Notes: This study used two subsets of the National Health Insurance Research Database: the Longitudinal Health Insurance Database [LHID] 2000 and LHID 2005 for vertical medical records from 1997 to 2012.

Abbreviation: LHID, Longitudinal Health Insurance Database.

reliable and an accurate proxy for date of mortality. ${ }^{12,13}$ Other minor situations to be withdrawn from the program, such as staying abroad, imprisonment, etc. Comorbidities were classified as diseases (eg, alcoholic fatty liver or hepatitis, CHB, $\mathrm{CHC}$, diabetes mellitus, hypertension, heart failure, and chronic kidney disease) existing prior to the index date.

\section{Outcome measure}

A diagnosis of liver cirrhosis was identified using ICD-9CM codes. Liver cirrhosis should meet one of the following enrollment criteria for inclusion in the study at least: 1) one or more inpatient admissions with major diagnosis of liver cirrhosis, or 2) at least three records of a liver cirrhosis diagnostic code made by a gastroenterologist specialist within 6 months. To increase the reliability of the liver cirrhosis diagnosis, we identified the patients who also received a liver ultrasound examination (NHI procedure code: 19001C or 19009C) during the study period. We also identified the liver complication diagnosis related to liver cirrhosis, such as hepatocellular carcinoma, variceal hemorrhage, ascites, and hepatic encephalopathy. Decompensated cirrhosis was defined as development of cirrhosis and encephalopathy, cirrhosis and ascites, or cirrhosis and variceal bleeding.

\section{Statistical analyses}

The baseline characteristics needed for data analysis of the two cohorts were identified from the insurance claim data files. These characteristics include age (40-50, 51-60, 61-70, and $>70$ years), gender, and medical history (with selected comorbidities). The number of liver cirrhosis developed among the enrolled subjects during the follow-up period was counted. Cox's regression hazard model was used to calculate the HR, whereas $P$-values were determined using log-rank test. Stratified Cox's regression hazard model was also adapted to compare the amiodarone cohort with the non-amiodarone cohort and thus evaluate the varying risks 
of developing liver cirrhosis. To determine the age effect on developing liver cirrhosis, we also conducted Cox's regression analysis by stratifying age into four groups (40-50, 51-60, 61-70, and $>70$ years). All data management and HR calculations were completed using the Statistical Analysis System (SAS) software for Windows (version 9.4; SAS Institute, Cary, NC, USA).

\section{Results}

\section{Characteristics of the study population}

The study included 8,081 patients in the amiodarone cohort; $55.55 \%$ were male, and the average age was 65.54 years (Table 1). The prevalent comorbidities in the amiodarone cohort were alcoholic fatty liver or hepatitis $(5.73 \%)$, CHB (1.47\%), CHC (1.58\%), diabetes (28.52\%), hypertension $(72.69 \%)$, and heart failure $(10.95 \%)$, while the prescribed medication on patients included cardiovascular drugs $(36.17 \%)$, diabetic drugs $(9.96 \%)$, statins $(8.13 \%)$, interferons, or nucleoside analogs for $\mathrm{CHB}$ treatment $(1.10 \%)$, and ribavirin and/or peginterferon for $\mathrm{CHC}$ treatment $(0.43 \%$ and $0.24 \%$, respectively). Among amiodarone cohort, $88.34 \%$ of subjects had received abdominal liver ultrasound examinations.

\section{The association between liver cirrhosis and amiodarone}

Before matching the data, the incidence of liver cirrhosis was significantly different between the two cohorts $(P=0.0338), 1.21 \%$ in the amiodarone cohort and $0.95 \%$ in the non-amiodarone cohort (Table 1). However, after adjustment the possible confounders, the amiodarone cohort had a nonsignificant risk of liver cirrhosis compared with the non-amiodarone cohort, with an adjusted HR (aHR) of 1.17 (95\% CI: 0.93-1.47; $P=0.1723$ ) (Table 2). The follow-up duration and cumulative incidences of cirrhosis for amiodarone and non-amiodarone cohorts are shown in Figure 2.

Table I The baseline and follow-up data of the study subjects

\begin{tabular}{|c|c|c|c|}
\hline & $\begin{array}{l}\text { Non-amiodarone } \\
(\mathrm{N}=32,324)\end{array}$ & $\begin{array}{l}\text { Amiodarone } \\
(\mathrm{N}=\mathbf{8}, \mathbf{0 8} \mathrm{I})\end{array}$ & $P$-value \\
\hline Age, $y$, mean $\pm S D$ & $66.31 \pm 11.56$ & $65.54 \pm I 1.64$ & $<0.0001$ \\
\hline Age group, n (\%) & & & 0.0001 \\
\hline $40-50$ & $3,273(10.13)$ & $916(11.34)$ & \\
\hline $5 I-60$ & $6,5 I I(20.14)$ & $1,715(21.22)$ & \\
\hline $6 I-70$ & $9,418(29.14)$ & $2,350(29.08)$ & \\
\hline$>70$ & $13,122(40.60)$ & $3,100(38.36)$ & \\
\hline Gender, n (\%) & & & $>0.9999$ \\
\hline Female & $14,368(44.45)$ & $3,592(44.45)$ & \\
\hline Male & $17,956(55.55)$ & $4,489(55.55)$ & \\
\hline Abdominal ultrasound, n (\%) & $25,018(77.40)$ & $7,139(88.34)$ & $<0.0001$ \\
\hline \multicolumn{4}{|l|}{ Medication, $\mathrm{n}(\%)$} \\
\hline Cardiovascular drugs & II,666 (36.09) & $2,923(36.17)$ & 0.8929 \\
\hline Diabetic drugs & $3,200(9.90)$ & $805(9.96)$ & 0.8678 \\
\hline Statins & $2,551(7.89)$ & $657(8.13)$ & 0.4787 \\
\hline \multicolumn{4}{|l|}{ Comorbidities, n (\%) } \\
\hline Alcoholic fatty liver or hepatitis & $\mathrm{I}, 480(4.58)$ & $463(5.73)$ & $<0.0001$ \\
\hline Chronic hepatitis B & $636(1.97)$ & $119(1.47)$ & 0.0033 \\
\hline Received I or $\mathrm{N}$ treatment $\mathrm{t}^{\mathrm{a}}$ & 451 (1.39) & $89(1.10)$ & 0.3515 \\
\hline Chronic hepatitis C & $510(1.58)$ & $128(1.58)$ & 0.9682 \\
\hline Received ribavirin treatment & $147(0.45)$ & $35(0.43)$ & 0.7957 \\
\hline Received peginterferon & $79(0.24)$ & $19(0.24)$ & 0.8801 \\
\hline Hypertension, n (\%) & $22,896(70.83)$ & $5,874(72.69)$ & 0.0010 \\
\hline Diabetes, n (\%) & $8,946(27.68)$ & $2,305(28.52)$ & 0.1284 \\
\hline Heart failure, n (\%) & $3,519(10.89)$ & $885(10.95)$ & 0.8669 \\
\hline Cirrhosis, n (\%) & $307(0.95)$ & $98(1.21)$ & 0.0338 \\
\hline Decompensated $^{\mathrm{b}}$ & $263(0.8 \mathrm{I})$ & $71(0.88)$ & 0.0104 \\
\hline Hepatocellular carcinoma & $122(0.38)$ & $22(0.27)$ & 0.0028 \\
\hline Variceal hemorrhage & $54(0.17)$ & $14(0.17)$ & 0.4904 \\
\hline Ascites & $70(0.22)$ & $25(0.3 \mathrm{I})$ & 0.5191 \\
\hline Hepatic encephalopathy & $233(0.72)$ & $59(0.73)$ & 0.0067 \\
\hline
\end{tabular}

Notes: al or $\mathrm{N}$ treatment denoted interferons and nucleoside analogs, including lamivudine, adefovir, telbivudine, and entecavir. ${ }^{\mathrm{b}}$ Decompensated liver cirrhosis indicated cirrhosis with any one of the followings: hepatocellular carcinoma, variceal hemorrhage, ascites, and hepatic encephalopathy. 
Table 2 Cox's regression hazards model analysis for prediction of occurrence of cirrhosis

\begin{tabular}{|c|c|c|c|c|}
\hline & \multirow{2}{*}{$\begin{array}{l}\text { Crude } \\
\text { HR }(95 \% \mathrm{CI})\end{array}$} & \multirow[t]{2}{*}{$P$-value } & \multirow{2}{*}{\begin{tabular}{|l|} 
Adjusted $^{\mathrm{a}}$ \\
HR $(95 \% \mathrm{CI})$
\end{tabular}} & \multirow[t]{2}{*}{$P$-value } \\
\hline & & & & \\
\hline Amiodarone & $\mathrm{I} .2 \mathrm{I}(0.97-\mathrm{I} .52)$ & 0.0975 & $1.17(0.93-1.47)$ & 0.1723 \\
\hline \multicolumn{5}{|l|}{ Comorbidities } \\
\hline Chronic hepatitis B & $3.54(2.32-5.39)$ & $<0.0001$ & $3.24(2.11-4.98)$ & $<0.0001$ \\
\hline Chronic hepatitis C & $9.60(7.06-13.06)$ & $<0.0001$ & $10.23(7.45-14.04)$ & $<0.000$ I \\
\hline Diabetes & I.I4 (0.92-I.4I) & 0.2315 & $0.64(0.5 \mathrm{I}-0.8 \mathrm{I})$ & 0.0002 \\
\hline Hypertension & $1.30(1.05-1.61)$ & 0.0169 & $0.76(0.54-1.05)$ & 0.0996 \\
\hline Heart failure & $1.64(1.23-2.19)$ & 0.0008 & $1.89(1.40-2.54)$ & $<0.0001$ \\
\hline \multicolumn{5}{|l|}{ Medications } \\
\hline Cardiovascular drugs & $6.13(4.89-7.70)$ & $<0.0001$ & $6.49(5.06-8.32)$ & $<0.000$ I \\
\hline Diabetic drugs & $3.47(2.76-4.36)$ & $<0.0001$ & $2.14(1.49-3.07)$ & $<0.000$ I \\
\hline Statins treatment & $1.28(0.9 \mathrm{I}-\mathrm{I} .8 \mathrm{I})$ & 0.1604 & $0.58(0.40-0.83)$ & 0.0031 \\
\hline
\end{tabular}

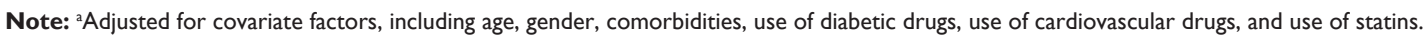

Significant differences were observed between amiodarone cohort and those non-amiodarone cohort in the liver cirrhosis-related decompensated status $(0.88 \%$ vs $0.81 \%$, $P=0.0104)$, such as hepatocellular carcinoma $(0.27 \%$ vs $0.38 \%, P=0.0028)$, variceal hemorrhage $(0.17 \%$ vs $0.17 \%$, $P=0.4904)$, ascites $(0.31 \%$ vs $0.22 \%, P=0.5191)$, and hepatic encephalopathy $(0.73 \%$ vs $0.72 \%, P=0.0067)$ (Table 1$)$.

\section{Comorbid disease status and the risk of liver cirrhosis}

The patients with hepatitis B were more likely to suffer from liver cirrhosis than those without hepatitis B in both cohorts
(Table S1) $(P<0.0001$, aHR: 3.24, 95\% CI: 2.11-4.98, Table 2). The patients with hepatitis $\mathrm{C}$ had a higher risk of liver cirrhosis than those without hepatitis $\mathrm{C}$ in both cohorts (Table S1) $(P<0.0001$, aHR: $10.23,95 \%$ CI: $7.45-14.04$, Table 2). The patients with heart failure had a higher risk of liver cirrhosis than those without heart failure in both cohorts (Table S1) $(P<0.0001$, aHR: $1.89,95 \%$ CI: $1.40-2.54$, Table 2). In addition, the patients receiving cardiovascular drugs or diabetic drugs were more likely to have liver cirrhosis than those without receiving cardiovascular drugs or diabetic drugs in both cohorts (Table S1) $(P<0.0001$, aHR: $6.49,95 \%$ CI: 5.06-8.32; $P<0.0001$, aHR: 2.14 ,

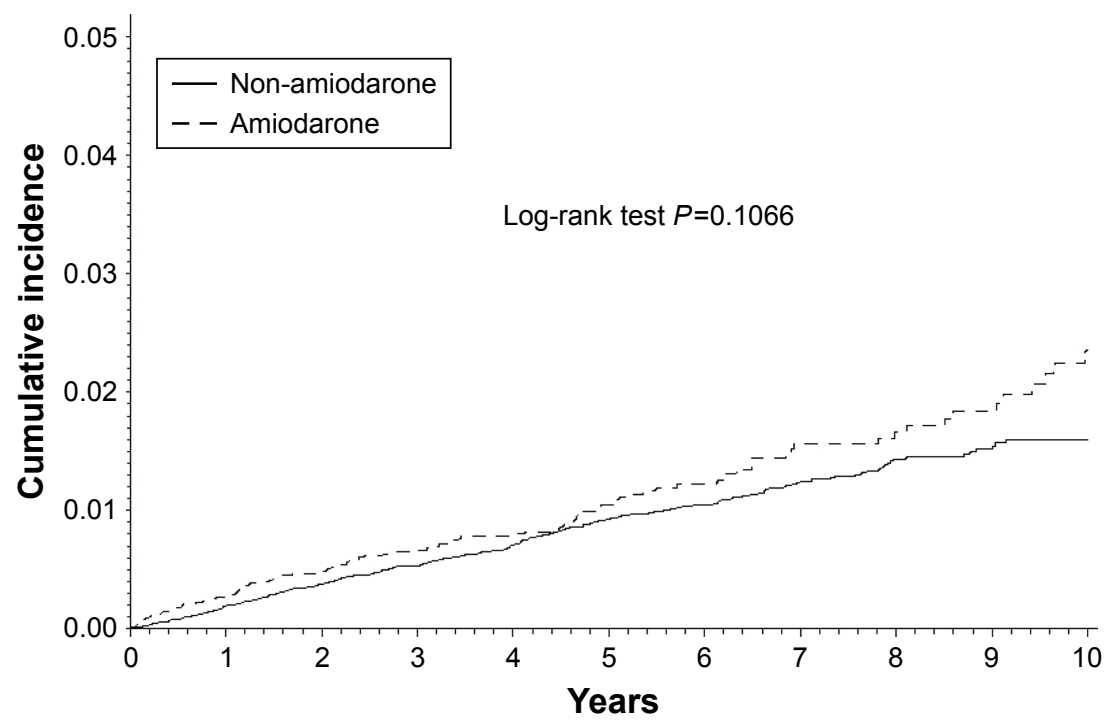

\begin{tabular}{lllllll}
\hline No at risk & Start & 2nd & 4th & 6th & 8th & 10th (years of follow-up) \\
\hline Non-amiodarone & 32,324 & 31,371 & 25,306 & 15,221 & 8,083 & 3,932 \\
Amiodarone & 8,081 & 7,664 & 6,208 & 4,147 & 2,529 & 1,371 \\
\hline
\end{tabular}

Figure 2 Cumulative incidences of liver cirrhosis in subjects with and without amiodarone.

Notes: Number of subjects at risk at the beginning and each year during the follow-up periods were shown in both cohorts. The number of subjects at risk decline over time due to death or data tracking time limit. "Start" refers to starting tracking; "2nd" refers to tracking the second year, and so on. 
Table 3 Subgroup analyses of cirrhosis risk in both cohorts: Cox's regression hazards model analysis for prediction of occurrence of cirrhosis in 40-50 age group

\begin{tabular}{|c|c|c|c|c|}
\hline & \multirow{2}{*}{$\begin{array}{l}\text { Crude } \\
\text { HR }(95 \% \text { CI) }\end{array}$} & \multirow[t]{2}{*}{$P$-value } & \multirow{2}{*}{\begin{tabular}{|l|l} 
Adjusted $^{\mathrm{a}}$ \\
HR $(95 \% \mathrm{CI})$ \\
\end{tabular}} & \multirow[t]{2}{*}{$P$-value } \\
\hline & & & & \\
\hline Amiodarone & I.2I (0.97-I.52) & 0.0975 & 1.17 (0.93-I.47) & 0.1723 \\
\hline \multicolumn{5}{|l|}{ Comorbidities } \\
\hline Chronic hepatitis B & $3.54(2.32-5.39)$ & $<0.0001$ & $3.24(2.11-4.98)$ & $<0.000$ I \\
\hline Chronic hepatitis C & $9.60(7.06-13.06)$ & $<0.000$ I & $10.23(7.45-14.04)$ & $<0.0001$ \\
\hline Diabetes & $1.14(0.92-1.41)$ & 0.2315 & $0.64(0.5 \mathrm{I}-0.8 \mathrm{I})$ & 0.0002 \\
\hline Hypertension & $1.30(1.05-1.61)$ & 0.0169 & $0.76(0.54-1.05)$ & 0.0996 \\
\hline Heart failure & $1.64(1.23-2.19)$ & 0.0008 & $1.89(1.40-2.54)$ & $<0.0001$ \\
\hline Cardiovascular drugs & $6.13(4.89-7.70)$ & $<0.0001$ & $6.49(5.06-8.32)$ & $<0.0001$ \\
\hline Diabetic drugs & $3.47(2.76-4.36)$ & $<0.000$ I & 2.14 (I.49-3.07) & $<0.0001$ \\
\hline Statins & $1.28(0.9 \mathrm{I}-\mathrm{I} .8 \mathrm{I})$ & 0.1604 & $0.58(0.40-0.83)$ & 0.0031 \\
\hline
\end{tabular}

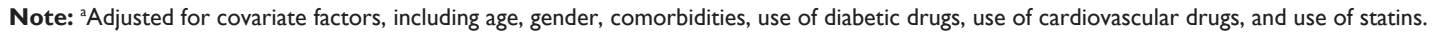

95\% CI: 1.49-3.07, respectively, Table 2). The patients receiving statin had a lower risk of liver cirrhosis than those without receiving statin $(P=0.0031$, aHR: $0.58,95 \% \mathrm{CI}$ : $0.40-0.83$, Table 2).

\section{Prediction for occurrence of liver cirrhosis in different age groups of patients}

To clarify the association between amiodarone and comorbidities and the risk of liver cirrhosis in different age groups of patients, we performed subgroup analyses of those aged 41-50, 51-60, 61-70, and >70 years, as shown in Tables 3-6. Using Cox's regression model for the sub-cohort analysis, we found that the patients receiving amiodarone aged 40-50, 51-60, 61-70, and $>70$ years had a nonsignificant risk of liver cirrhosis than those without receiving amiodarone after the data were adjusted $(P=0.1723$, aHR: $1.17,95 \%$ CI: $0.93-1.47$; $P=0.8815$, aHR: $0.94,95 \% \mathrm{CI}: 0.40-2.20 ; P=0.1053$,
aHR: $1.35,95 \%$ CI: 0.94-1.95; $P=0.1415$, aHR: 1.32 , 95\% CI: 0.91-1.92). The patients with hepatitis B aged 40-50 and 61-70 years had a higher risk of liver cirrhosis than those without hepatitis $\mathrm{B}$ after the data were adjusted $(P<0.0001$, aHR: 3.24, 95\% CI: 2.11-4.98; $P<0.0001$, aHR: 4.01, 95\% CI: 2.09-7.68). The patients with hepatitis $\mathrm{C}$ aged 40-50, 61-70 and $>70$ years had a higher risk of liver cirrhosis than those without hepatitis $\mathrm{C}$ after the data were adjusted $(P<0.0001$, aHR: 10.23, 95\% CI: 7.45-14.04; $P<0.0001$, aHR: 9.17, 95\% CI: 5.44-15.45; $P<0.0001$, aHR: 15.32, 95\% CI: 9.44-24.85). The patients with heart failure aged 40-50 and 61-70 years had a higher risk of liver cirrhosis than those without heart failure after the data were adjusted $(P<0.0001$, aHR: $1.89,95 \%$ CI: $1.40-2.54$; $P<0.0001$, aHR: 3.06 , 95\% CI: 1.90-4.92). The patients receiving diabetes drugs aged $40-50$ and $61-70$ years had a higher risk of liver cirrhosis than those without receiving diabetes drugs after the data were adjusted $(P<0.0001$,

Table 4 Subgroup analyses of cirrhosis risk in both cohorts: Cox's regression hazards model analysis for prediction of occurrence of cirrhosis in $51-60$ age group

\begin{tabular}{l|l|l|l|l}
\hline & Crude & P-value & Adjusted $^{\text {a }}$ & P-value \\
\cline { 4 - 5 } & HR (95\% Cl) & & HR (95\% Cl) & \\
\hline Amiodarone & $1.03(0.44-2.40)$ & 0.9446 & $0.94(0.40-2.20)$ & 0.8815 \\
Comorbidities & & & \\
$\quad$ Chronic hepatitis B & $1.57(0.21-11.65)$ & 0.6563 & $2.02(0.27-15.12)$ & 0.4957 \\
Chronic hepatitis C & $5.79(0.78-42.71)$ & 0.0852 & $5.92(0.8-43.89)$ & 0.0819 \\
Diabetes & $1.66(0.79-3.50)$ & 0.1846 & $1.18(0.52-2.67)$ & 0.6943 \\
Hypertension & NA & & NA & \\
Heart failure & NA & & NA & \\
Cardiovascular drugs & $3.37(1.65-6.92)$ & 0.0009 & $3.31(1.5-7.34)$ & 0.0031 \\
Diabetic drugs & NA & & NA & \\
Statins & $1.74(0.24-12.76)$ & 0.5876 & $1.19(0.16-9.1)$ & 0.8669 \\
\hline
\end{tabular}

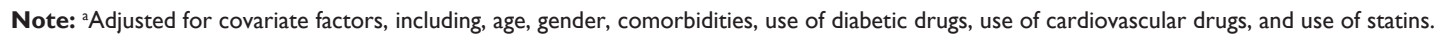
Abbreviation: NA, not available. 
Table 5 Subgroup analyses of cirrhosis risk in both cohorts: Cox's regression hazards model analysis for prediction of occurrence of cirrhosis in $61-70$ age group

\begin{tabular}{|c|c|c|c|c|}
\hline & \multirow{2}{*}{$\begin{array}{l}\text { Crude } \\
\text { HR }(95 \% \mathrm{Cl})\end{array}$} & \multirow[t]{2}{*}{$P$-value } & \multirow{2}{*}{$\begin{array}{l}\text { Adjusted }^{\mathrm{a}} \\
\text { HR }(95 \% \mathrm{CI})\end{array}$} & \multirow[t]{2}{*}{$P$-value } \\
\hline & & & & \\
\hline Amiodarone & $1.39(0.96-2.00)$ & 0.0779 & $1.35(0.94-1.95)$ & 0.1053 \\
\hline \multicolumn{5}{|l|}{ Comorbidities } \\
\hline Chronic hepatitis B & $3.46(1.82-6.59)$ & 0.0002 & $4.01(2.09-7.68)$ & $<0.000$ I \\
\hline Chronic hepatitis C & $7.83(4.72-13.00)$ & $<0.0001$ & $9.17(5.44-15.45)$ & $<0.000$ I \\
\hline Diabetes & $0.95(0.66-1.36)$ & 0.7682 & $0.58(0.40-0.85)$ & 0.0057 \\
\hline Hypertension & $1.28(0.91-1.80)$ & 0.1575 & $0.67(0.40-1.13)$ & 0.1346 \\
\hline Heart failure & $2.32(1.46-3.69)$ & 0.0004 & $3.06(1.90-4.92)$ & $<0.000$ I \\
\hline Cardiovascular drugs & $5.13(3.52-7.47)$ & $<0.0001$ & $5.14(3.39-7.81)$ & $<0.000$ I \\
\hline Diabetic drugs & $3.49(2.45-4.99)$ & $<0.0001$ & $2.44(1.39-4.30)$ & 0.0020 \\
\hline Statins & $1.66(1.04-2.63)$ & 0.0329 & $0.84(0.51-1.37)$ & 0.4739 \\
\hline
\end{tabular}

Note: aAjusted for covariate factors, including age, gender, comorbidities, use of diabetic drugs, use of cardiovascular drugs, and use of statins.

aHR: $2.14,95 \%$ CI: $1.49-3.07 ; P=0.0020$, aHR: $2.44,95 \%$ CI: 1.39-4.30). The patients receiving cardiovascular drugs aged $40-50,51-60,61-70,>70$ years had a higher risk of liver cirrhosis than those without receiving cardiovascular drugs after the data were adjusted $(P<0.0001$, aHR: 6.49 , 95\% CI: 5.06-8.32; $P=0.0031$, aHR: 3.31, 95\% CI: $1.5-7.34$; $P<0.0001$, aHR: 5.14, 95\% CI: 3.39-7.81; $P<0.0001$, aHR: 10.43 , 95\% CI: 6.73-16.18). In addition, the patients receiving statin aged 40-50 and 61-70 years had a lower risk of liver cirrhosis than those without receiving statin after the data were adjusted ( $P=0.0031$, aHR: $0.58,95 \% \mathrm{CI}$ : $0.40-0.83$; $P=0.0235$, aHR: $0.48,95 \%$ CI: $0.26-0.91)$.

\section{Discussion}

Our main finding showed that liver cirrhosis incidence was higher in the amiodarone cohort without a high risk of liver cirrhosis than in the non-amiodarone cohort $(1.21 \%$ vs
$0.95 \% ; P=0.030$, Table 1), with an aHR of 1.16 (95\% CI $0.93-1.44 ; P=0.1856$, Table 2), after adjusting for possible confounders. Amiodarone induces liver cirrhosis, which is an uncommon complication of chronic oral amiodarone therapy. ${ }^{14,15}$ With the progress of heart failure treatment and increasing aging population, many elderly patients receive amiodarone therapy due to its irreplaceable role in left ventricular dysfunction. Cardiohepatic interaction in heart failure results in cardiogenic liver injury on drug metabolism. ${ }^{16}$ However, large-scale studies about this important issue were lacking. The present study included a largescale cohort (identified 8,081 cases in the amiodarone cohort) with a long follow-up time (up to 10 years). We excluded liver cirrhosis diagnosis in both cohorts prior to this study and included several important comorbidities possibly related to liver cirrhosis. Our result concurred with that of Mattar et al, ${ }^{17}$ who examined the use of amiodarone in a population of

Table 6 Subgroup analyses of cirrhosis risk in both cohorts: Cox's regression hazards model analysis for prediction of occurrence of cirrhosis in $>70$ age group

\begin{tabular}{|c|c|c|c|c|}
\hline & \multirow{2}{*}{\begin{tabular}{|l|} 
Crude \\
HR $(95 \% \mathrm{Cl})$ \\
\end{tabular}} & \multirow[t]{2}{*}{$P$-value } & Adjusted $^{a}$ & \multirow[t]{2}{*}{$P$-value } \\
\hline & & & HR (95\% Cl) & \\
\hline Amiodarone & $1.32(0.9 \mid-1.91)$ & 0.1487 & $1.32(0.91-1.92)$ & 0.1415 \\
\hline \multicolumn{5}{|l|}{ Comorbidities } \\
\hline Chronic hepatitis B & $4.18(1.55-11.29)$ & 0.0048 & $2.56(0.91-7.15)$ & 0.0734 \\
\hline Chronic hepatitis C & $12.06(7.60-19.14)$ & $<0.0001$ & $15.32(9.44-24.85)$ & $<0.0001$ \\
\hline Diabetes & $0.7 \mid(0.49-1.03)$ & 0.0682 & $0.40(0.27-0.59)$ & $<0.0001$ \\
\hline Hypertension & $1.17(0.84-1.65)$ & 0.3508 & $1.02(0.62-1.68)$ & 0.9317 \\
\hline Heart failure & $1.07(0.72-1.60)$ & 0.7242 & $1.27(0.84-1.92)$ & 0.2531 \\
\hline Cardiovascular drugs & $8.75(5.77-13.27)$ & $<0.0001$ & $10.43(6.73-16.18)$ & $<0.0001$ \\
\hline Diabetic drugs & $3.02(2.08-4.38)$ & $<0.0001$ & I.46 (0.84-2.56) & 0.1833 \\
\hline Statins & $1.06(0.58-1.97)$ & 0.8422 & $0.48(0.26-0.91)$ & 0.0235 \\
\hline
\end{tabular}

Note: adjusted for covariate factors, including age, gender, comorbidities, use of diabetic drugs, use of cardiovascular drugs, and use of statins. 
409 patients with metabolic syndrome or heart failure. They found no cases of clinical hepatitis, cirrhosis, or death related to the therapeutic use of amiodarone in that population during an average observation period of 3 years. ${ }^{17}$ In our amiodarone cohort, $10.95 \%$ (885 cases) had heart failure, $28.52 \%$ had type 2 diabetes mellitus (T2DM), 72.69\% had hypertension, and 3.05\% had CHB and CHC. Our result showed that chronic amiodarone use did not significantly increase the risk of liver cirrhosis across the different age groups. On a population basis, properly managed amiodarone use had no excess risk of liver cirrhosis compared with patients without receiving amiodarone. With the prevalence of abnormal transaminases in the population, a baseline measurement is a key to interpret whether transaminase levels increase in response to amiodarone. Furthermore, given the slow change in amiodarone exposure with changing dose and patient environment, determining ALT change every 3 months during the first year should be adequate to identify toxicity issues. Later, adequate testing should be conducted every 6 months. Accurate downward titration to the lowest effective dose is also important for any drug, including amiodarone. ${ }^{18}$

Another important finding was that heart failure patients with a high risk of developing liver cirrhosis showed an aHR of 1.89 in all study subjects (95\% CI: $1.40-2.54 ; P<0.0001$, Table 2). These observations were significant in younger patients (aged 40-50 and 61-70 years) with a high risk of liver cirrhosis and with an aHR of 1.89 and $3.06(95 \%$ CI: $1.40-2.54 ; P<0.0001$, Table 3; 95\% CI: 1.90-4.92; $P<0.0001$, Table 5, respectively), but not in patients older than 70 years. This result highlighted the importance of cardiohepatic interaction in heart failure patients, which is less described clinically. Furthermore, heart failure patients may present liver-related symptoms, including abdominal distention, intermittent right upper quadrant discomfort, nausea, early satiety, or anorexia. ${ }^{16}$ These patients may be indistinguishable from patients with liver disease or heart failure due to potential subtle presentations of symptoms. These findings may occur in the absence of overt ascites or lower extremity edema (particularly in younger patients). Thus, high-degree awareness about heart failure-related cardiohepatic interactions should be addressed by physicians to decrease heart failure-associated morbidity and mortality. ${ }^{7,16}$ We suggest that heart failure patients with abnormal liver function should first be evaluated for potential biliary tract obstruction and/or primary hepatic pathology before attributing irregularities to cardiac disease.

The present study revealed that patients with $\mathrm{CHB}$ and $\mathrm{CHC}$ were at a high risk for developing liver cirrhosis (aHR: 3.24, 95\% CI: 2.11-4.98, $P<0.0001$; aHR: 10.23,
95\% CI: 7.45-14.04; $P<0.0001$, respectively). Our results agree with the previous finding that $\mathrm{CHB}$ and $\mathrm{CHC}$ are the leading causes of chronic liver disease, especially cirrhosis. ${ }^{19}$ Subgroup analysis in different age groups showed that $\mathrm{CHC}$ in advanced age may accelerate fibrosis progression. The aHR of CHC for liver cirrhosis in ages more than 71 years was higher than that in the younger age groups (aged 40-50 and 61-70 years). Individuals with HCV infection are often asymptomatic and unaware of their illness until severe liver diseases occur and progress to liver cirrhosis. ${ }^{20}$ This observation may be the reason why patients with a high $\mathrm{HR}$ of $\mathrm{CHC}$ are at risk of liver cirrhosis. In addition, the present study found that patients receiving diabetic drugs were more likely to have liver cirrhosis than those without receiving diabetic drugs $(P<0.0001$, aHR: $2.14,95 \% \mathrm{CI}$ : 1.49-3.07, respectively). Previous studies have shown that non-alcoholic fatty liver disease (NAFLD) and T2DM are common conditions that regularly co-exist and can act synergistically to drive adverse outcomes. ${ }^{21}$ Furthermore, in a series of 432 patients with biopsy-proven NAFLD, the presence of co-existent T2DM may increase the risk of NAFLD progression to fibrosis. ${ }^{22}$ Our results agree with the previous finding that T2DM is an independent risk factor for fibrosis. Furthermore, the use of statins was associated with a significant $42 \%$ reduction in the risk of cirrhosis, which is consistent with the finding of a recent meta-analysis about statins and the risk of cirrhosis in $\mathrm{CHB}$ or $\mathrm{CHC}$ patients. ${ }^{23}$ However, in the present study, these protective effects were more evident in age 40-50 and 61-70 years than in other age groups ( $P=0.0031$, aHR: $0.58,95 \%$ CI: $0.40-0.83 ; P=0.0235$, aHR: 0.48, 95\% CI: 0.26-0.91, respectively, Tables 3-6).

\section{Limitations}

This study exhibits several limitations. First, the diagnosis of liver cirrhosis was identified through ICD-9 codes, instead of confirmatory liver biopsy. The validity of the ICD-9 codes to define cirrhosis was unknown. However, in daily practice, liver biopsy is rarely pursued by health providers. To increase the accuracy of the diagnosis of cirrhosis, we enrolled patients with at least three records of a liver cirrhosis diagnostic code made by a gastroenterologist within 6 months, or one or more inpatient admissions with diagnosis of liver cirrhosis. In the present study, $88.34 \%$ of patients among amiodarone cohort and $77.40 \%$ of patients among non-amiodarone cohort had received liver ultrasound examinations. Furthermore, the agreement between the NHIRD and the self-reported data of 15,660 subjects ranged between $82.5 \%$ and $97.3 \% .{ }^{24}$ Although through the administrative database, patients' data are anonymized, we cannot identify 
the symptoms of decompensated liver cirrhosis and cannot precisely determine the severity of liver cirrhosis, but we can identify the complications of liver cirrhosis as a proxy of disease severity. In the present study, around $80 \%$ of cirrhosis patients were with related complications. Second, in the present study, we did not have information regarding a patient's HBV viral load or liver function. However, Taiwan's NHI program has strict regulations regarding reimbursement for nucleoside analogs. Only high-risk populations, including patients with higher baseline HBV viral load, higher serum ALT level or higher prevalence of liver decompensation are eligible for reimbursement. In our study, $74 \%$ of CHB patients in amiodarone cohort vs $70 \%$ of CHB patients in non-amiodarone cohort were eligible for treatment reimbursement $(P=0.3515)$. In addition, the criteria for anti$\mathrm{HCV}$ therapy reimbursement are seropositivity for anti-HCV and HCV RNA for $>6$ months with an elevated ALT level. In our study, $42 \%$ of $\mathrm{CHC}$ patients in amiodarone cohort vs $44 \%$ of $\mathrm{CHC}$ patients in non-amiodarone cohort were eligible for treatment reimbursement. These data provided us information on the proportion of $\mathrm{HCV}$ viremic patients with abnormal liver function in both cohorts. Third, the database lacks personal information, which may affect cirrhosis risk, such as lifestyle, body weight, body mass index, smoking habits, and alcohol use information. Fourth, this study had a retrospective cohort design, and the results are prone to inherent selection bias. Further prospective studies are necessary to confirm our observations.

\section{Conclusion}

In the Taiwanese population, patients in the amiodarone cohort without a high risk of liver cirrhosis were compared with patients in the non-amiodarone cohort. Patients with specific comorbid diseases, including T2DM, CHB, CHC, and heart failure, were probably at a high risk of developing liver cirrhosis. Long-term surveillance for liver toxicity in high-risk patients with amiodarone treatment was suggested. The use of statins was associated with a significant $42 \%$ reduction in the risk of liver cirrhosis.

\section{Disclosure}

The authors report no conflicts of interest in this work.

\section{References}

1. Conde D, Costabel JP, Alves de Lima A. Recent-onset atrial fibrillation in patients with left ventricular dysfunction: amiodarone or vernakalant? Can J Cardiol. 2013;29(10):1330.e11-1330.e12.

2. Cadrin-Tourigny J, Wyse DG, Roy D, et al. Efficacy of amiodarone in patients with atrial fibrillation with and without left ventricular dysfunction: a pooled analysis of AFFIRM and AF-CHF trials. J Cardiovasc Electrophysiol. 2014;25(12):1306-1313.
3. Goldschlager N, Epstein AE, Naccarelli GV, et al; Practice Guidelines Sub-committee, North American Society of Pacing and Electrophysiology (HRS). A practical guide for clinicians who treat patients with amiodarone: 2007. Heart Rhythm. 2007;4(9):1250-1259.

4. Kirchhof P, Benussi S, Kotecha D, et al; ESC Scientific Document Group. 2016 ESC Guidelines for the management of atrial fibrillation developed in collaboration with EACTS. Eur Heart J. 2016;37(38):2893-2962.

5. Chiang CE, Wu TJ, Ueng KC, et al. 2016 Guidelines of the Taiwan Heart Rhythm Society and the Taiwan Society of Cardiology for the management of atrial fibrillation. J Formos Med Assoc. 2016;115(11): 893-952.

6. Fang JC, Ewald GA, Allen LA, et al; Heart Failure Society of America Guidelines Committee. Advanced (stage D) heart failure: a statement from the Heart Failure Society of America Guidelines Committee. J Card Fail. 2015;21(6):519-534.

7. Pendyal A, Gelow JM. Cardiohepatic Interactions: implications for management in advanced heart failure. Heart Fail Clin. 2016;12(3): 349-361.

8. Hussain N, Bhattacharyya A, Prueksaritanond S. Amiodarone-induced cirrhosis of liver: what predicts mortality? ISRN Cardiol. 2013;2013: 617943 .

9. LiverTox website. Bethesda, MD: National Institutes of Health; 2018 [updated 2018 April 18; cited 2018 April 20]; Drug Record: Amiodarone. Available from: https://ivertox.nlm.nih.gov/Amiodarone.htm

10. Wu CY, Chen YJ, Ho HJ, et al. Association between nucleoside analogues and risk of hepatitis B virus-related hepatocellular carcinoma recurrence following liver resection. JAMA. 2012;308(18):1906-1914.

11. Yu ML, Yeh ML, Tsai PC, et al. Huge gap between clinical efficacy and community effectiveness in the treatment of chronic hepatitis $\mathrm{C}$ : a nationwide survey in Taiwan. Medicine (Baltimore). 2015;94(13):e690.

12. Lien HM, Chou SY, Liu JT. Hospital ownership and performance: evidence from stroke and cardiac treatment in Taiwan. J Health Econ. 2008;27(5):1208-1223.

13. Cheng CL, Chien HC, Lee CH, Lin SJ, Yang YH. Validity of in-hospital mortality data among patients with acute myocardial infarction or stroke in National Health Insurance Research Database in Taiwan. Int J Cardiol. 2015;201:96-101.

14. Atiq M, Davis JC, Lamps LW, Beland SS, Rose JE. Amiodarone induced liver cirrhosis. Report of two cases. J Gastrointestin Liver Dis. 2009; 18(2):233-235.

15. Puli SR, Fraley MA, Puli V, Kuperman AB, Alpert MA. Hepatic cirrhosis caused by low-dose oral amiodarone therapy. Am J Med Sci. 2005;330(5):257-261.

16. Samsky MD, Patel CB, Dewald TA, et al. Cardiohepatic interactions in heart failure: an overview and clinical implications. J Am Coll Cardiol. 2013;61(24):2397-2405.

17. Mattar W, Juliar B, Gradus-Pizlo I, Kwo PY. Amiodarone hepatotoxicity in the context of the metabolic syndrome and right-sided heart failure. J Gastrointestin Liver Dis. 2009;18(4):419-423.

18. Pollak PT. How toxic is amiodarone to the liver? J Gastrointestin Liver Dis. 2010;19(1):11-13.

19. Liaw YF, Chu CM. Hepatitis B virus infection. Lancet. 2009; 373(9663):582-592.

20. Lee MH, Yang HI, Yuan Y, L'Italien G, Chen CJ. Epidemiology and natural history of hepatitis C virus infection. World J Gastroenterol. 2014;20(28):9270-9280.

21. Hazlehurst JM, Woods C, Marjot T, Cobbold JF, Tomlinson JW. Nonalcoholic fatty liver disease and diabetes. Metabolism. 2016;65(8): 1096-1108.

22. Hossain N, Afendy A, Stepanova M, et al. Independent predictors of fibrosis in patients with nonalcoholic fatty liver disease. Clin Gastroenterol Hepatol. 2009;7(11):1224-1229.

23. Wang Y, Xiong J, Niu M, et al. Statins and the risk of cirrhosis in hepatitis B or C patients: a systematic review and dose-response meta-analysis of observational studies. Oncotarget. 2017;8(35):59666-59676.

24. Yu ST, Chang HY, Lin MC, Lin YH. Agreement between self-reported and health insurance claims on utilization of health care: a population study. J Clin Epidemiol. 2009;62(12):1316-1322. 


\section{Supplementary material}

Table S I Cox's regression hazards model analysis for prediction of occurrence of cirrhosis in amiodarone cohort and non-amiodarone cohort

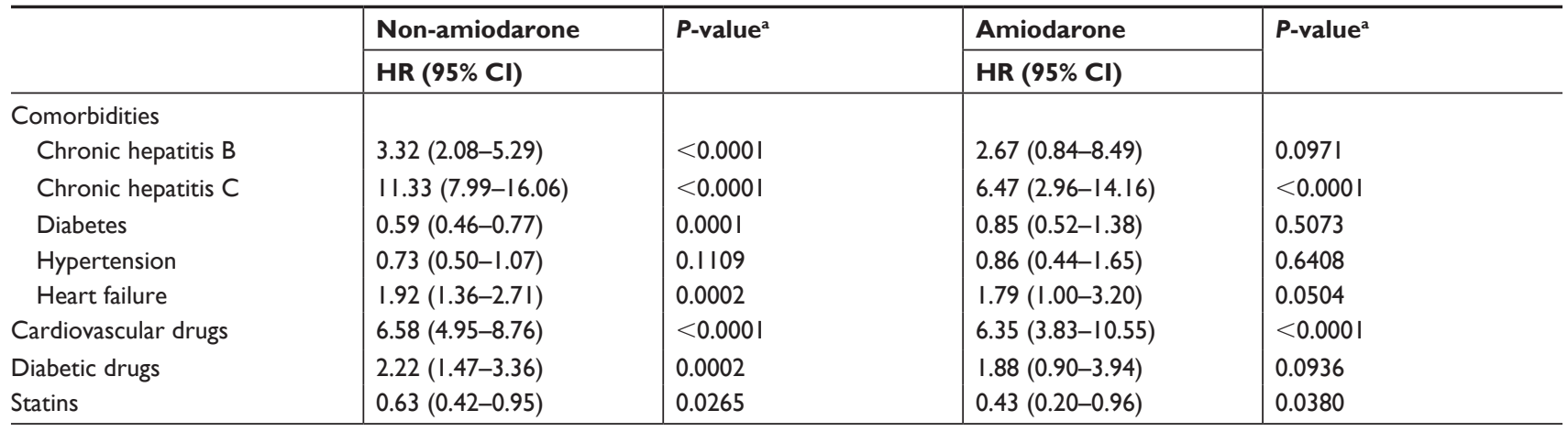

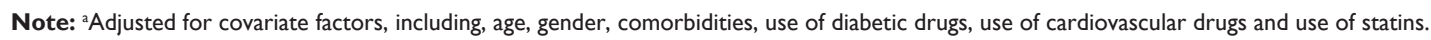

\section{Publish your work in this journal}

Therapeutics and Clinical Risk Management is an international, peerreviewed journal of clinical therapeutics and risk management, focusing on concise rapid reporting of clinical studies in all therapeutic areas, outcomes, safety, and programs for the effective, safe, and sustained use of medicines. This journal is indexed on PubMed Central, CAS,
EMBase, Scopus and the Elsevier Bibliographic databases. The manuscript management system is completely online and includes a very quick and fair peer-review system, which is all easy to use. Visit http://www.dovepress.com/testimonials.php to read real quotes from published authors. 